\title{
Real-Time Strain Mapping via Biaxial Stretching in Heart Valve Tissues
}

\author{
Hsiao-Ying Shadow Huang* and Siyao Huang
}

\begin{abstract}
Previous studies show that the collagen fiber architecture is key to the heart valves tissue mechanical property. We report a real-time strain mapping approach that provides displacement vectors and principal strain directions during the mechanical characterization of heart valve tissues. The strain maps reported in the current study allows an individual to quickly identify the approximate strain imposed on a location of the sample. The result shows that when samples are biaxially stretched under $18 \%$ strain, less anisotropy is observed in both aortic and pulmonary valve leaflet samples. Moreover, when samples are stretched from $\mathbf{2 8 \%}$ to $35 \%$, pulmonary valves leaflet samples exhibits a stronger anisotropic effect than aortic valve. Therefore, a higher degree of straightening is required for collagen fibers to be fully aligned. This work provides an easy approach to quantify mechanical properties with the corresponding strain maps of heart valve tissues and potentially facilitates the developments of tissue engineering heart valves.
\end{abstract}

\section{INTRODUCTION}

Heart valve leaflets are thin, collagenous tissues whose anisotropic biomechanical function depends intimately on biochemical composition and structure [1]. In states of disease, the leaflets can become too stiff or too floppy. A common theme, however, in both stenotic and myxomatous disease is disturbance of the fine-tuned structure-function relations governing heart valve homeostasis [2]. Toward understanding the valves and how best to replace them, numerous studies have focused on characterizing their mechanical behavior [3-5], microstructural organization [6, 7], and molecular and cellular level compositions [8-10]. However, while their essential anatomic peculiarities have been acknowledged for nearly a century [11], heart valve leaflets continue to challenge investigators, in particular in the context of tissue engineering [12].

Tissue engineering approaches aim to generate living, native-like prosthetics from combinations of cells and biodegradable polymer scaffolds [13]. The motivation for heart valve tissue engineering is manifold, as current mechanical and bioprosthetic replacement heart valves, while lifesaving, manifest a host of deficiencies and complications. Paramount perhaps are the implications for pediatric patients, for whom the lack of growth potential characteristic of current non-viable valve replacements mandates periodic

This work is support by Faculty Research and Professional Development Grant at North Carolina State University. Asterisk indicates corresponding author.

*HY S. Huang is with the Department of Mechanical Engineering, North Carolina State University, Raleigh, NC 27695 USA (email: hshuang@ncsu.edu).

S. Huang is with the Department of Mechanical Engineering, North Carolina State University, Raleigh, NC 27695 USA (email: shuang4@ncsu.edu). reoperation with associated risks of morbidity and mortality [14]. Introduced by Shinoka et al. [15], heart valve tissue engineering has since invoked the full gamut of available technologies, ranging from noninvasively accessible stem cells [16] to pulsatile flow loop bioreactors [17]. Indeed, while superficially simple, heart valve leaflets are complex tissues whose characterization, let alone recapitulation, has historically relied on mastery of specialized biochemical assays and mechanical test equipment.

Hallmarks of heart valve leaflets are their nonlinear, anisotropic mechanical response and web-like collagen network. While their prototypical soft tissue stress-strain curves - comprised of toe, transition, and linear regions - can be obtained by conventional uniaxial tensile testing [18], formulation of structural-based constitutive models amenable to simulating in situ deformation has traditionally relied on biaxial testing [19]. In particular, biaxial tensile testing data retain the influence of axial coupling, allowing for more precise predictions under generalized loading conditions. Biaxial testing devices, however, are generally custom-built and significantly more complex than their uniaxial counterparts, including computer-coordinated control of two loading axes and optical mapping of tissue strains [20]. For the heart valve tissue engineer, a relatively simple, commercially available biaxial tester could facilitate testing and accelerate development.

The BioTester 5000 (CellScale Biomaterials Testing, Waterloo, Ontario) was introduced in 2008 as a turnkey biaxial test system [21]. Designed for testing planar tissues and biomaterials under physiologic conditions, the BioTester has previously been applied to materials ranging from human sclera [22] to annulus fibrosa [23]. In the current study, a BioTester was used to characterize the biaxial mechanical response of porcine aortic (AV) and pulmonary valve (PV) leaflet tissues, providing a comparison with previously reported data, and establishing a basis for future applications.

\section{MATERIALS AND METHODS}

In brief, nine porcine hearts from large sows (greater than $300 \mathrm{lbs}$ ) are obtained from the Nahunta Pork Center (Pikeville, NC) immediately after slaughtering and are returned to the laboratory within 60 minutes of sacrifice for dissection. The AV and PV are dissected from each heart, and the leaflets are removed from their respective roots. The removal is completed by cutting axially along aorta and pulmonary artery toward the heart until the valves are reached. Each leaflet is then stretched out and held taut while being cut against the wall of the artery. Each of twelve AV and PV leaflet samples $(\sim 7-\mathrm{mm} \times 7-\mathrm{mm})$ are marked according to the Cartesian coordinate system: $x$-axis represents the circumferential direction and $y$-axis represents 
the radial direction of the heart valve tissues. Three dots are marked with a surgical marker after the samples are cut to size: one of the dots acting as the origin and the other two made to define each axis. The samples are stored in Hank's Balance Salt Solution (HBSS) for the relaxation. A biaxial tissue tester (BioTester 5000, CellScale, Waterloo, Canada), equipped with two load cells $(500 \mathrm{mN} \pm 1 \mathrm{mN})$ for each axis of loading, is used for measuring the mechanical properties of semilunar heart valve tissue leaflets. Synchronized time lapse video for real-time monitoring and post process analysis is provided by the charged-couple device (CCD) camera, which acquires images with a pixel resolution of $1280 \times 960$ at an acquisition rate of $15-\mathrm{Hz}$, with lens focal length as 75-mm (Fig. 1a). A temperature controlled saline bath with data logging capability provides a physiological environment for testing soft tissue specimen.

The specimen mounting method is considered as one of the major challenges of biaxial testing [21]. For example, artifacts such as suturing procedures usually cause discrepancy results due to inconsistent boundary conditions. In contrast, BioRakes provides fast and accurate sample mounting: each BioRake consists of five tungsten tins used to anchor one edge of the specimen [21] (Fig. 1b). Four rakes provide uniform attachment across the edge of the samples and evenly distributed loading spanning 4-mm in length on each side of the sample. This unique feature not only assures the control of boundary conditions, but also reduces significantly the variability between sample sizes. That is, if the sample size prepared is larger than $4-\mathrm{mm}^{2}$, the active loading area remains $4-\mathrm{mm} \times 4-\mathrm{mm}$. After the sample is mounted, the sample is lowered into the HBSS bath that is heated to $37^{\circ} \mathrm{C}$ as in-vivo physiological environment. The heart valve leaflet samples are tested up to $35 \%$ strain on both axes with a 15 second stretch, a 15 second recovery, with no hold time. The imaging tracking and analysis software (LabJoy, CellScale, Waterloo, Canada) are used to review and analyze images collected during our biaxial testing (Fig. 1c).

\section{RESUltS AND DiscusSION}

\section{A. Mechanical Properties of Heart Valves}

After averaging over measured stress vs. strain of AV and PV leaflets samples under equi-biaxial testing, the result is shown in Fig. 2. The plot shows the correlation between the strain and the resulting stress in the circumferential (xaxis) and radial (y-axis) directions for both $\mathrm{AV}$ and $\mathrm{PV}$ samples. The means \pm standard deviations biaxial stiffness values of AV and PV samples are listed in Fig. 2. In Fig. 2, three zones are observed: zone 1 is between $0 \%$ to $18 \%$ strain stretching, zone 2 is between $18 \%$ to $28 \%$ strain stretching, and zone 3 is between $28 \%$ to $35 \%$ strain stretching. The difference of the direction-dependent stress vs. strain curves in AV and PV samples is mainly due to collagen fibers arrangements. Most of collagen fibers in AV and PV align circumferentially, therefore stiffer mechanical property in the $\mathrm{x}$-direction is observed. Since collagen fibers in PV are more randomly distributed comparing to that in AV, AV samples exhibit stronger anisotropic material behavior than that of PV samples.

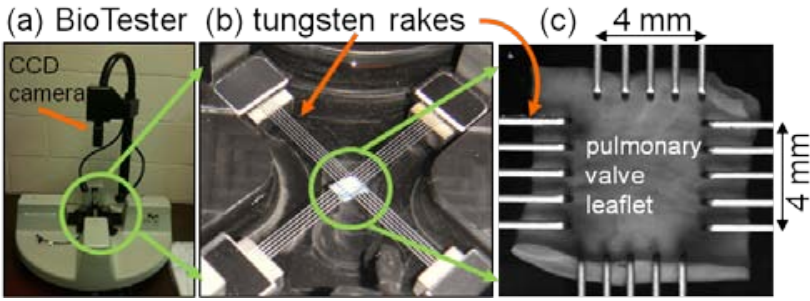

Fig.1. The BioTester is capable of applying physiologically plausible biaxial loading states to tissue samples. The tungsten rakes pierce through samples and provide evenly distributed loading. This unique feature assures the control of loading conditions and significantly reduces the variability associated with testing different sample sizes.

\section{B. Mechanical Behaviors and the Relations to the Collagen Fiber Microstructures}

In zone $1(0-18 \%$ strain $)$, it is observed that the stiffness in the $\mathrm{x}$-direction is more than twice of that in the $\mathrm{y}$-direction for AV samples $(89.1 \pm 4.63 \mathrm{kPa}: 33.94 \pm 1.37 \mathrm{kPa} \approx 2.6)$, suggesting AV has a slightly anisotropy materials property in zone 1. In contrast, the stiffness is comparable in $\mathrm{x}-$ and $\mathrm{y}-$ directions for PV samples $(11.31 \pm 0.79 \mathrm{kPa}: 11.67 \pm 0.61 \mathrm{kPa}$ $\approx 1$ ), suggesting that $\mathrm{PV}$ exhibits isotropic material property in zone 1 . It could be due to that randomly distributed collagen fibers in PV samples are not fully aligned along with the circumferential direction before reaching $18 \%$ biaxial stretching. The result also shows that AV samples is more than 7 times stiffer than that of PV samples in the x-direction $(89.1 \pm 4.63 \mathrm{kPa}: 11.31 \pm 0.79 \mathrm{kPa} \approx 7.8)$, but in the $\mathrm{y}-$ direction, AV samples is only 3 times stiffer than that of PV samples $(33.94 \pm 1.37 \mathrm{kPa}: 11.67 \pm 0.61 \mathrm{kPa} \approx 3)$. It is suggested that collagen fibers for AV samples align with the circumferential direction sooner than that in the PV samples before reaching $18 \%$ biaxial stretching.

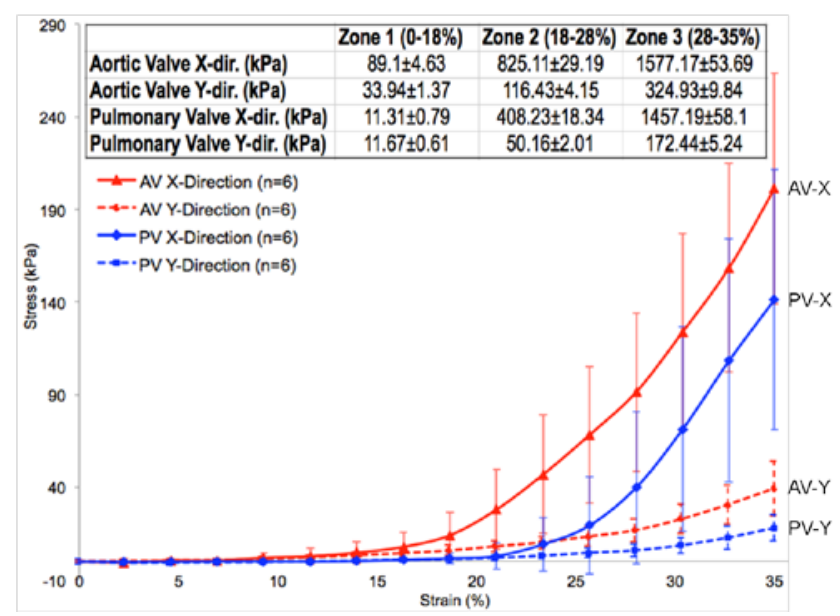

Fig. 2. Mechancial properties for AV and PV tissue samples. A nonlinear anisotropic mechanical property is observed in both AV and PV tissue samples. Three zones are observed: zone 1 (0-18\% strain), zone 2 (18-28\% strain), and zone 3 (28-35\% strain).

In zone $2(18-28 \%$ strain), it is observed that the stiffness in the $\mathrm{x}$-direction is more than 7 times higher than that in the $\mathrm{y}$-direction for both AV and PV samples $(825.11 \pm 29.19 \mathrm{kPa}$ : $116.43 \pm 4.15 \mathrm{kPa} \approx 7$ and $408.23 \pm 18.34 \mathrm{kPa}: 50.16 \pm 2.01 \mathrm{kPa}$ $\approx 8$, respectively). This finding is comparable to ones reported by Lewinsohn et al. via uniaxial tensile testing [3] and correlates well with the range of previous mechanical 
characterization studies via force-control biaxial testing [24]. The similar $\mathrm{x}$ to $\mathrm{y}$ ratio of mechanical property observed in zone 2 suggests that the distribution of collagen fibers along in the circumferential direction comparing to that along in the radial direction is comparable in both $\mathrm{AV}$ and $\mathrm{PV}$ samples: the degree of anisotropy of fiber distribution is similar. AV samples is twice stiffer than that of PV samples $(825.11 \pm 29.19 \mathrm{kPa}(\mathrm{AV} ; \mathrm{x}): 408.23 \pm 18.34 \mathrm{kPa}(\mathrm{PV} ; \mathrm{x}) \approx 2$ and $116.43 \pm 4.15 \mathrm{kPa}(\mathrm{AV} ; \mathrm{y}): 50.16 \pm 2.01 \mathrm{kPa} \approx 2$ (PV; y)) in the both $\mathrm{x}$ and $\mathrm{y}$ directions, and it is suggested that collagen fibers exhibit similar architectures in the AV and PV samples. However, it is still not clear which intrinsic biological characteristic gives rise to the interesting microstructure features of collagen fibers reflecting back to the measured mechanical property in zone 2 .

In zone 3 (28-35\% strain), it is observed that the stiffness in the $\mathrm{x}$-direction is more than four times higher than that in the y-direction for AV samples $(1577.17 \pm 53.69 \mathrm{kPa}$ : $324.93 \pm 9.84 \mathrm{kPa}=4.85$ ), where the $\mathrm{x}$ to $\mathrm{y}$ ratio is lower than that in zone $2(825.11 \pm 29.19 \mathrm{kPa}: 116.43 \pm 4.15 \mathrm{kPa}=7)$. The decrease $\mathrm{x} / \mathrm{y}$ ratio of mechanical property in AV samples suggests that collagen fibers experience most rearrangement and straightening in zone 2 and exhibit a linear elastic manner in zone 3. Different results are observed for PV samples and indicate that the stiffness in the $\mathrm{x}$-direction is more than 8 times higher than that in the $y$-direction $(1457.19 \pm 58.1 \mathrm{kPa}: 172.44 \pm 5.24 \mathrm{kPa} \approx 8.5)$, and the result has the similar $\mathrm{x} / \mathrm{y}$ ratio as observed in zone $2(408.23 \pm 18.34$ $\mathrm{kPa}: 50.16 \pm 2.01 \mathrm{kPa} \approx 8.13)$. It is suggested that collagen fibers exhibit higher anisotropy in PV than that in AV in zone 3. It could be due to that collagen fibers are highly random distributed in the PV leaflets than that in the AV leaflet. Therefore, a higher degree of straightening is required for collagen fibers to be fully aligned. The stiffness is similar in the $\mathrm{x}$-direction for both AV $(1577.17 \pm 53.69 \mathrm{kPa})$ and $\mathrm{PV}$ samples $(1457.19 \pm 58.1 \mathrm{kPa})$, but $\mathrm{AV}(324.93 \pm 9.84 \mathrm{kPa})$ is almost twice stiffer than that of PV $(172.44 \pm 5.24 \mathrm{kPa})$ in the $\mathrm{y}$-direction.

Comparing to the result in zone 1, a almost ten-fold increase in the stiffness in the $\mathrm{x}$-direction for AV samples is observed $(825.11 \pm 29.19 \mathrm{kPa}$ (zone $2 ; \mathrm{x}): 89.1 \pm 4.63 \mathrm{kPa}$ (zone $1 ; \mathrm{x}) \approx 9.3$ ), where a substantial increase $(\sim 36$ times $)$ in the stiffness in the $\mathrm{x}$-direction for $\mathrm{PV}$ samples is measured $(408.23 \pm 18.34 \mathrm{kPa}$ (zone $2 ; \mathrm{x}): 11.31 \pm 0.79 \mathrm{kPa}$ (zone $1 ; \mathrm{x}) \approx$ 36). In contrast, only three- and four-fold increase in the stiffness in the y-direction for both $\mathrm{AV}$ and $\mathrm{PV}$ samples is observed (116.43 $\pm 4.15 \mathrm{kPa}$ (AV; zone 2, y): $33.94 \pm 1.37 \mathrm{kPa}$ $(\mathrm{AV}$; zone $1 ; \mathrm{y}) \approx 3.43$ and $50.16 \pm 2.01 \mathrm{kPa}$ (PV; zone 2, y): $11.67 \pm 0.61 \mathrm{kPa}(\mathrm{PV}$; zone $1, \mathrm{y}) \approx 4$, respectively). The result confirms that highly anisotropic materials property in heart valve tissues is dominated by the orientation of circumferential aligned collagen fibers [7,24,25]. Moreover, collagen fibers in PV samples exhibit stronger realignment beyond $18 \%$ strain and the materials exhibit anisotropically rather than isotropically as ones observed in zone 1 .

Comparing the ratios in zone $2(825.11 \pm 29.19 \mathrm{kPa}(\mathrm{AV}$; $\mathrm{x}): 408.23 \pm 18.34 \mathrm{kPa}(\mathrm{PV} ; \mathrm{x}) \approx 2$ and $116.43 \pm 4.15 \mathrm{kPa}(\mathrm{AV}$; y) : $50.16 \pm 2.01 \mathrm{kPa} \approx 2(\mathrm{PV} ; \mathrm{y}))$, the decreased ratio between samples in zone 3 is observed. Moreover, the stiffness of AV and PV samples in both $\mathrm{x}$ - and $\mathrm{y}$-directions are consistently more than twice of ones in zone $2(1577.17 \pm 53.69 \mathrm{kPa}(\mathrm{AV}$; zone $3 ; \mathrm{x})$ : $825.11 \pm 29.19 \mathrm{kPa}(\mathrm{AV} ;$ zone $2 ; \mathrm{x}) \approx 2$,

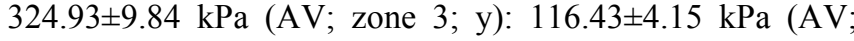
zone $2 ; \mathrm{y}) \approx 2.8,1457.19 \pm 58.1 \mathrm{kPa}(\mathrm{PV}$; zone $3 ; \mathrm{x})$ : $408.23 \pm 18.34 \mathrm{kPa}(\mathrm{PV}$; zone $2 ; \mathrm{x}) \approx 3.6$, and $172.44 \pm 5.24$ $\mathrm{kPa}(\mathrm{PV}$; zone 3 ; y) : 50.16 $\pm 2.01 \mathrm{kPa} \approx 3.4$ (PV; zone $2 ; \mathrm{y})$ ), suggesting that both $\mathrm{AV}$ and $\mathrm{PV}$ tissue samples exhibit the collagen fiber alignment is saturated when samples are stretched above $28 \%$ strain. The consistent higher mechanical property in the $\mathrm{x}$-direction for AV samples could contribute to the quantity of collagen fibers. The higher mechanical property in the $y$-direction for AV samples could be explained by the amount of collagen fibers cross-linking [26].

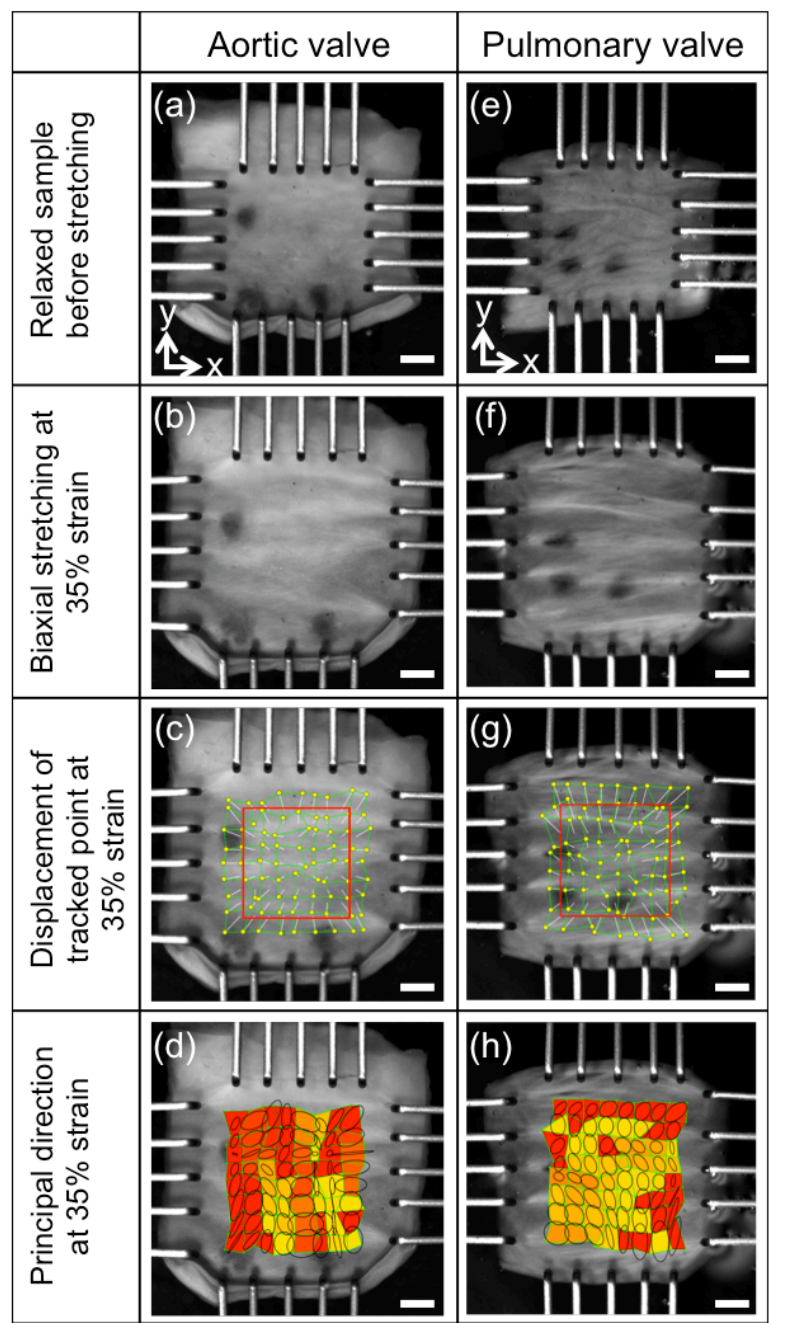

Figure 3. Corresponding macroscopical appearance of AV and PV samples under equibiaxial testing. Maps of Displacement and principal strain are shown at 35\% equibiaxial stretching. Collagen bundles are observed in PV samples. Scale bar: 1-mm.

\section{Real-time strain maps in heart valve tissues}

Corresponding macroscopical appearance of AV and PV samples under equibiaxial testing is captured via an integrated image analysis module of Biotester. Motion of image features, such as specimen texture, fiducial markers, are recorded and used to study localized AV and PV sample deformations, strain magnitudes, and principal axes (Fig. 3). Relaxed AV and PV samples before the equibiaxial testing are shown in Fig. 3(a) and (e), whereas four rakes provide 
evenly distributed loading spanning $4 \mathrm{~mm}$ in length on each side of the sample. Fig. 3(b) and (f) show that AV and PV samples are stretched up to $35 \%$ equilbiaxially. PV leaflets are generally thinner than AV leaflets $(0.38-\mathrm{mm}$ and $0.56-$ $\mathrm{mm}$ respectively). Therefore, under equibiaxial testing, fiber bundles parallel to the circumferential direction in the PV samples are shown clearly than ones in the AV samples. Displacements of tracked points under equibiaxial 35\% strain are shown in Fig. 3(c) and (g). The red boxes define the reference containing eighty-one points at $0 \%$ strain. The yellow dots indicate the locations of eighty-one points when samples are biaxially stretched at $35 \%$. The green tails indicate the relative displacements of tracked points from ones at previous strain $(\sim 32 \%)$ strain. The map of principal axes for deformed AV and PV samples at 35\% equibiaxial stretch is showed in Fig. 3(d) and (h). The principal axes are shown in black circles, and they are averaged between 16 points via the integrated image analysis module. Color red in the map indicates higher value of principal axis, and yellow indicates a lower principal axis value.

\section{CONCLUSION}

The current study provides a simple approach to investigate mechanical properties of heart valve tissues with the corresponding displacement vectors and principal strain maps. The maps reported in the current study allows an individual to quickly identify the approximate strain imposed on a location of the sample, as well as to detect any potential grip effects that generally cause discrepancy results due to inconsistent boundary conditions. From the measured stress-strain curves, three zones are observed. In zone 1 (0$18 \%$ strain), AV has a slightly anisotropic material property, however, PV exhibits an isotropic material property. In zone 2 (18-28\% strain), the distribution of collagen fibers along the circumferential direction versus that along the radial direction is comparable in both AV and PV samples, suggesting that collagen fibers exhibit similar arrangements in the AV and PV samples. In zone 3 (28-35\% strain), collagen fibers exhibit higher anisotropy in PV than that in AV. Moreover, the stiffness of AV and PV samples in both the circumferential and radial directions are consistently two- and three-fold higher than ones in zone 2, suggesting that collagen fiber alignment in both AV and PV tissues is saturated when samples are stretched above $28 \%$ strain.

\section{REFERENCES}

[1] M.S. Sacks, W.D. Merryman, and D.E. Schmidt, "On the biomechanics of heart valve function," Journal of Biomechanics, vol. 42, (no. 12), pp. 1804-1824, 2009.

[2] F.J. Schoen, "Mechanisms of function and disease of natural and replacement heart valves," Annual review of pathology, vol. 7, pp. 161-83, Feb 282012.

[3] A.D. Lewinsohn, A. Anssari-Benham, D.A. Lee, P.M. Taylor, A.H. Chester, M.H. Yacoub, and H.R.C. Screen, "Anisotropic strain transfer through the aortic valve and its relevance to the cellular mechanical environment," Proceedings of the Institution of Mechanical Engineers Part H-Journal of Engineering in Medicine, vol. 225, (no. H8), pp. 821-830, 2011.

[4] T.M. Koch, B.D. Reddy, P. Zilla, and T. Franz, "Aortic valve leaflet mechanical properties facilitate diastolic valve function RID C-3386-2009," Computer methods in biomechanics and biomedical engineering, vol. 13, (no. 2), pp. 225-234, 2010.

[5] J.A. Stella and M.S. Sacks, "On the biaxial mechanical properties of the layers of the aortic valve leaflet," Journal of Biomechanical Engineering-Transactions of the Asme, vol. 129, (no. 5), pp. 757-766, 2007.
[6] T.C. Doehring, M. Kahelin, and I. Vesely, "Mesostructures of the aortic valve," Journal of Heart Valve Disease, vol. 14, (no. 5), pp. 679-686, 2005.

[7] E.M. Joyce, J. Liao, F.J. Schoen, J.E. Mayer, Jr., and M.S. Sacks, "Functional Collagen Fiber Architecture of the Pulmonary Heart Valve Cusp RID F-3703-2011," Annals of thoracic surgery, vol. 87, (no. 4), pp. 1240-1249, 2009.

[8] N. Latif, R. Sarathchandra, R.M. Taylor, J. Antoniw, and M.H. Yacoub, "Molecules mediating cell-ECM and cell-cell communication in human heart valves," Cell biochemistry and biophysics, vol. 43, (no. 2), pp. 275-287, 2005.

[9] H.-Y.S. Huang, J. Liao, and M.S. Sacks, "In-situ deformation of the aortic valve interstitial cell nucleus under diastolic loading," Journal of Biomechanical Engineering, vol. 129, (no. Journal Article), pp. 1-10, 2007. [10] D.A. Filip, A. Radu, and M. Simionescu, "Interstitial-Cells of the Heart-Valves Possess Characteristics Similar to Smooth-Muscle Cells," Circulation research, vol. 59, (no. 3), pp. 310-320, 1986.

[11] L. Gross and M.A. Kugel, "Topographical anatomy and histology of the valves in the human heart," Am J Pathol, vol. 7, (no. Journal Article), pp. 225-276, 1931.

[12] M.S. Sacks, F.J. Schoen, and J.E. Mayer, Jr., "Bioengineering Challenges for Heart Valve Tissue Engineering," ANNUAL REVIEW OF BIOMEDICAL ENGINEERING, vol. 11, (no. Journal Article), pp. 289-313, 2009.

[13] R. Langer and J.P. Vacanti, "Tissue engineering," Science, vol. 260, (no. 5110), pp. 920-6, 1993.

[14] J. Perron, A.M. Moran, K. Gauvreau, P.J. del Nido, J.E. Mayer, and R.A. Jonas, "Valved homograft conduit repair of the right heart in early infancy," Annals of thoracic surgery, vol. 68, (no. 2), pp. 542-548, 1999.

[15] T. Shinoka, C.K. Breuer, R.E. Tanel, G. Zund, T. Miura, P.X. Ma, R. Langer, J.P. Vacanti, and J.E. Mayer, Jr., "Tissue engineering heart valves: valve leaflet replacement study in a lamb model," Ann Thorac Surg, vol. 60, (no. 6 Suppl), pp. S513-6, Dec 1995.

[16] F. Colazzo, P. Sarathchandra, R.T. Smolenski, A.H. Chester, Y.-T. Tseng, J.T. Czernuszka, M.H. Yacoub, and P.M. Taylor, "Extracellular matrix production by adipose-derived stem cells: Implications for heart valve tissue engineering," Biomaterials, vol. 32, (no. 1), pp. 119-127, 2011. [17] J.L. Berry, J.A. Steen, J.K. Williams, J.E. Jordan, A. Atala, and J.J. Yoo, "Bioreactors for Development of Tissue Engineered Heart Valves," Annals of Biomedical Engineering, vol. 38, (no. 11), pp. 3272-3279, 2010.

[18] A. Sauren, M.C. Hout, A.A. Steenhaven, F.E. Veldpaus, and J.D. Janssen, "The Mechanical Properties of Porcine Aortic Valve Tissue," Journal of Biomechanics, vol. 16, (no. Journal Article), pp. 327-337, 1983.

[19] M.S. Sacks, "Incorporation of experimentally-derived fiber orientation into a structural constitutive model for planar collagenous tissues," $J$ Biomech Eng, vol. 125, (no. 2), pp. 280-7, 2003.

[20] J.S. Grashow, M.S. Sacks, J. Liao, and A.P. Yoganathan, "Planar biaxial creep and stress relaxation of the mitral valve anterior leaflet," Annals of Biomedical Engineering, vol. 34, (no. 10), pp. 1509-1518, 2006.

[21] A. Eilaghi, J.G. Flanagan, G.W. Brodland, and C.R. Ethier, "Strain Uniformity in Biaxial Specimens is Highly Sensitive to Attachment Details," Journal of Biomechanical Engineering-Transactions of the Asme, vol. 131, (no. 9), pp. 091003-091003, 2009.

[22] A. Eilaghi, J.G. Flanagan, I. Tertinegg, C.A. Simmons, G.W. Brodland, and C.R. Ethier, "Biaxial mechanical testing of human sclera," Journal of Biomechanics, vol. 43, (no. 9), pp. 1696-1701, 2010.

[23] V. Etacheri, R. Marom, R. Elazari, G. Salitra, and D. Aurbach, "Challenges in the development of advanced Li-ion batteries: a review," Energy \& Environmental Science, vol. 4, (no. 9), pp. 3243-3262, 2011.

[24] K.L. Billiar and M.S. Sacks, "Biaxial mechanical properties of the natural and glutaraldehyde treated aortic valve cusp - Part I: Experimental results," Journal of Biomechanical Engineering-Transactions of the Asme, vol. 122, (no. 1), pp. 23-30, 2000.

[25] G.W. Christie and B.G. Barratt-Boyes, "Mechanical Properties of Porcine Pulmonary Valve Leaflets: How Do They Differ from Aortic Leaflets?," Ann Thorac Surg, vol. 60, (no. 0003-4975), pp. S195-S199, 1995.

[26] A. Balguid, M.P. Rubbens, A. Mol, R.A. Bank, A.J.J.C. Bogers, J.P. Van Kats, B.A.J.M. De Mol, F.P.T. Baaijens, and C.V.C. Bouten, "The role of collagen cross-links in biomechanical behavior of human aortic heart valve leaflets - Relevance for tissue engineering," Tissue engineering, vol. 13, (no. 7), pp. 1501-1511, 2007. 